\title{
A novel approach to study radiation track structure with nanometer-equivalent resolution ${ }^{\star}$
}

\author{
Margherita Casiraghi ${ }^{1, a}$, Vladimir Bashkirov ${ }^{2}$, Ford Hurley ${ }^{2}$, and Reinhard Schulte ${ }^{2,3}$ \\ 1 Center for Proton Therapy, Paul Scherrer Institut, 5232 Villigen PSI, Switzerland \\ 2 Department of Basic Sciences, Loma Linda University, 11175 Campus St., Loma Linda, CA 92350, USA \\ 3 Department of Radiation Medicine, Loma Linda University Medical Center, 11234 Anderson St., Loma Linda, \\ CA 92354, USA
}

Received 31 December 2013 / Received in final form 8 March 2014

Published online 7 May 2014 - (c) EDP Sciences, Società Italiana di Fisica, Springer-Verlag 2014

\begin{abstract}
Clustered DNA damages are considered the critical lesions in the pathways leading from the initial energy deposition by radiation to radiobiological damage. The spatial distribution of the initial DNA damage is mainly determined by radiation track-structure at the nanometer level. In this work, a novel experimental approach to image the three-dimensional structure of micrometric radiation track segments is presented. The approach utilizes the detection of single ions created in low-pressure gas. Ions produced by radiation drift towards a GEM-like 2D hole-pattern detector. When entering individual holes, ions can induce ion-impact ionization of the working-gas starting a confined electron avalanche that generates the output signal. By registering positive ions rather than electrons, diffusion is reduced and a spatial resolution of the track image of the order of water-equivalent nanometers can be achieved. Measurements and simulations to characterize the performance of a few detector designs were performed. Different cathode materials were tested and ionization cluster size distributions of ${ }^{241} \mathrm{Am}$ alpha particles were measured. The electric field configuration in the detector was calculated to optimize the ion focusing into the detector holes. The preliminary results obtained show the directions for further development of the detector.
\end{abstract}

\section{Introduction}

The biological effects of ionizing radiation are primarily defined by the spatial distribution of ionization events relative to sensitive biological targets. The DNA molecule, a structure of $2 \mathrm{~nm}$ in transverse diameter, is believed to be the most important target. Monte Carlo simulations of radiation track structure have shown that a wide range of ionization cluster sizes is produced in DNA-like targets, with large clusters occurring at higher frequencies for densely ionizing radiations [1]. Generally, a higher frequency of large ionization clusters will translate in a higher frequency of clustered DNA damages at the end of the chemical phase. The exact relation between ionization clusters and DNA damage clusters depends on the amount of radical recombination and on repair mechanisms. Clustered DNA damages are considered the critical lesions leading to permanent biological damage.

The experimental characterization of the ionization patterns is useful for monitoring of mixed or unknown radiation fields and it is needed for benchmarking Monte

\footnotetext{
* Contribution to the Topical Issue "Nano-scale Insights into Ion-beam Cancer Therapy", edited by Andrey V. Solov'yov, Nigel Mason, Paulo Limão-Vieira and Malgorzata SmialekTelega.

a e-mail: margherita.casiraghi@psi.ch
}

Carlo simulation codes. In the nanodosimetric approach, such characterization is performed by measuring ionization cluster size distributions (ICSDs) produced by radiation interacting with low-pressure gas. Monte Carlo simulations show that, when properly scaled, the ICSDs measured in macroscopic gas volumes are approximately equivalent to the ICSDs created in nanometric volumes of water [2]. The scaling factor that has to be applied to the gas measurements depends on the gas density and on the ratio of the ionization mean free path length of the primaries in the two media.

Only a few nanodosimeters have been developed so far [3]. The measuring method of these devices is based on the detection of single ionizations in a gas of low pressure, simulating target volumes of about $1 \mu \mathrm{g} \mathrm{cm}^{-2}$ mass per area [4]. The ion-counting nanodosimeter [5] and the jet-counter [6] measure the ICSD created in a sensitive volume simulating a short segment of DNA by detecting positive ions. A different approach is used in the tracknanodosimetric counter [7], which detects electrons produced in single ionizations and it is able to measure the ionization cluster formation in nanometric-equivalent volumes as a function of distance from the primary particle path.

In this work, we present the characterization of a novel nanodosimeter able to image the entire three-dimensional structure of the radiation track with single-ion resolution. 


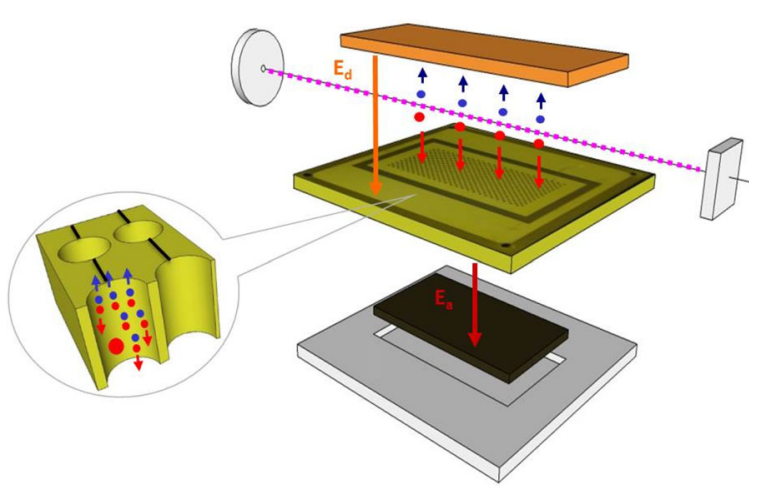

Fig. 1. Schematic representation of the detector design. From top to bottom: copper anode providing a drift field $\left(E_{d}\right)$ through the sensitive volume, PCB board with $2 \mathrm{D}$ hole-array and readout electrode, high-resistivity cathode providing the accelerating field $\left(E_{a}\right)$, Teflon ${ }^{\circledR}$ layer for cathode insulation. The enlarged view shows the signal generation principle.

The detector design was initially presented by Bashkirov et al. [8]. With this device, ionization patterns produced by arbitrary radiation fields interacting with the working gas can be registered. The detector combines the working principle of thick gas electron multipliers (THGEM) [9] working in reverse polarity and of resistive plate chambers [10]. THGEM-based multipliers are capable of registering ionizing particles and photons with high efficiency through charge multiplication - initiated by secondary electrons - in the detector working gas. The use of electrodes of highly resistive bulk material provides confinement of the multiplication avalanche. For nanodosimetry applications, ionization events have to be localized with nanometer-equivalent resolution. This level of resolution can be achieved by registering ions rather than electrons. Precise localization of electrons is indeed compromised by ballistic motion and larger diffusion compared to ions. In order to detect low energy ions, at least one free electron must be created by ion-impact ionization in one of the detector holes. This is achieved by working in low pressure and at high electric fields.

In this work, simulations and measurements testing this detector principle have been performed. Different detector prototypes were assembled and their performance assessed and compared.

\section{Material and methods}

\subsection{Detector principle and design}

The principal detector design is presented in Figure 1. The main detector component is a copper-clad dielectric printed circuit board (PCB) of about $3 \mathrm{~mm}$ thickness with a two-dimensional array of sub-millimeter diameter holes. The bottom side of the board is in contact with a highresistivity cathode, which is connected to the negative high voltage $(\mathrm{HV})$ providing the accelerating field. In the latest design, the top side of the PCB provides three readout strip layers that are connected to ground by a $50 \Omega$

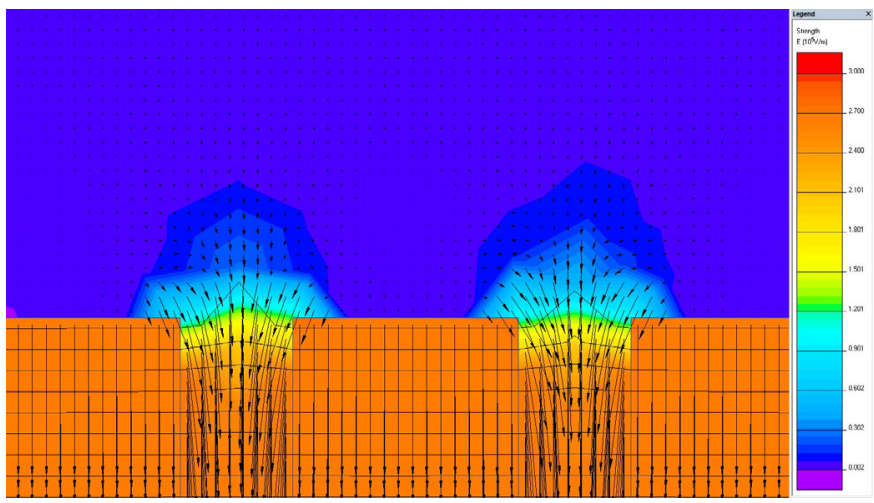

Fig. 2. Color-map representation of the electric field strength, in a vertical section of the detector, calculated with the QuickField software. The electric field lines are represented by arrows. The calculation was performed for a drift field of $20 \mathrm{~V} \mathrm{~cm}^{-1}$, an $\mathrm{HV}$ of $-800 \mathrm{~V}$ and grounded readout strips.

load and separated by $127 \mu \mathrm{m}$ of dielectric. The central and the bottom layers are formed by orthogonal strips common to each row and column of holes, respectively providing 2D readout of individual holes. The top layer of strips, oriented at 45 degrees with respect to the other two layers, was added to resolve hit ambiguities.

The ionizing radiation traversing the working gas volume above the detector produces electron-ion pairs. Electrons are collected by the anode while ions drift towards the detector plane under the influence of a drift field of the order of tens of $\mathrm{V} \mathrm{cm}^{-1}$. As shown in Figure 2, the accelerating field provided by the cathode penetrates into the drift region, providing the focusing of the ions into the holes of the PCB. Operating at gas pressure of the order of hundreds of Pascals $(\mathrm{Pa})$, the reduced electric field inside each hole is of the order of $10 \mathrm{~V} \mathrm{~cm}^{-1} \mathrm{~Pa}^{-1}$. Under such conditions, the cross-section for ion-impact ionization is higher than under standard pressure conditions and may approach $100 \%$ for a hole height of the order of few millimeters.

The secondary electron produced in the ion-impact ionization initiates a charge avalanche which propagates towards the top of the hole and induces a signal in the readout strips. The avalanche is spatially confined to the hole by the dielectric walls of the PCB and it stops when the voltage across the hole drops due to the high-volume resistivity of the cathode. Therefore, each hole operates as an independent Geiger counter.

Due to the readout strip configuration, the $2 \mathrm{D}$ position of the holes containing the avalanche can be reconstructed by using a multichannel data acquisition system. In the left panel of Figure 3, a prototype of the detector connected to $3 \times 24$ readout channels is shown. Because the ion diffusion in the working gas is small compared to the size of the holes, the coordinates of the holes containing the avalanche correspond to the $2 \mathrm{D}$ position of initial ionization. Therefore, the output of the detector comprises a series of $2 \mathrm{D}$ projections of the ionizing particle track. The complete $3 \mathrm{D}$ track is then reconstructed by measuring the time difference between detector signals. 


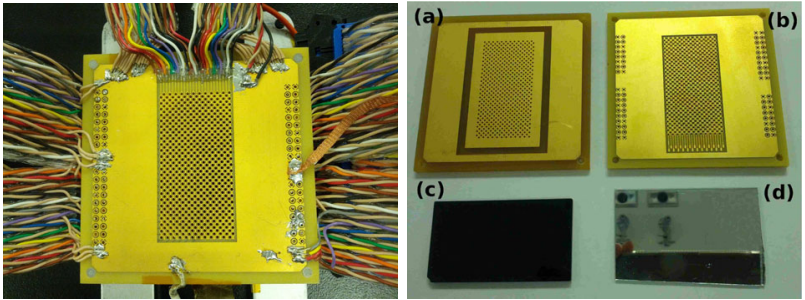

Fig. 3. Left panel: Assembled detector prototype connected to the readout electronics with $3 \times 24$ channels. Right panel: $\mathrm{PCBs}$ and cathodes combined in the different detector prototypes. (a) PCB with $0.8 \mathrm{~mm}$ hole diameter and common top electrode; (b) PCB with $1 \mathrm{~mm}$ hole diameter and three layers of readout strips. Both PCBs have an active area of $2 \times 5 \mathrm{~cm}^{2}$. (c) Semiconductor cathode; (d) high-resistivity glass cathode.

\subsection{Experimental set-up}

We tested the operational principle of the detector with different prototypes (Fig. 3). The first PCB layout had a common top electrode while the latest design has threelayers as described above. Each PCB type was combined with either a high-resistivity glass cathode or a semiconductor cathode. The top surface of the cathode was in contact with the working gas only through the detector holes. The bottom side of the cathode, connected to the HV, was carefully insulated from the working gas to prevent uncontrolled discharges. A copper anode was placed above the detector plane and connected to a positive power supply providing the drift voltage. The prototypes were installed into a low-pressure gas chamber providing a controlled gas pressure. For all experiments, propane was used as working gas at a pressure ranging from $133 \mathrm{~Pa}$ to $667 \mathrm{~Pa}$. This corresponded to a tissue equivalent volume up to few tens of $\mu \mathrm{m}$.

A $2 \mathrm{~mm}$ collimated beam of ${ }^{241} \mathrm{Am}$ alpha particles placed $5 \mathrm{~mm}$ above the detector plane was used as primary radiation field. The alpha particles traversing the volume were detected with a photo-diode placed on the opposite side of the source, beyond the ion detector. The photo-diode signal was used as trigger to start the data acquisition window of $600 \mu \mathrm{s}$ of duration. The detector signal and the trigger signal were acquired by a dedicated data acquisition system providing amplification, discrimination and analysis of the signals. The data analysis was performed with a field-programmable gate array (FPGA) programmed to count the number of pulses per trigger and to measure the pulse arrival time with respect to the trigger.

The HV applied to the cathode ranged from $-650 \mathrm{~V}$ to $-900 \mathrm{~V}$; the optimal value was chosen according to the gas pressure to obtain the highest registration efficiency. The drift field ranged from $5 \mathrm{~V} \mathrm{~cm}^{-1}$ to $20 \mathrm{~V} \mathrm{~cm}^{-1}$, not exceeding the threshold for electron multiplication.

\section{Results and discussion}

The signal measured with the detector prototype utilizing the PCB with common top electrode and the high-

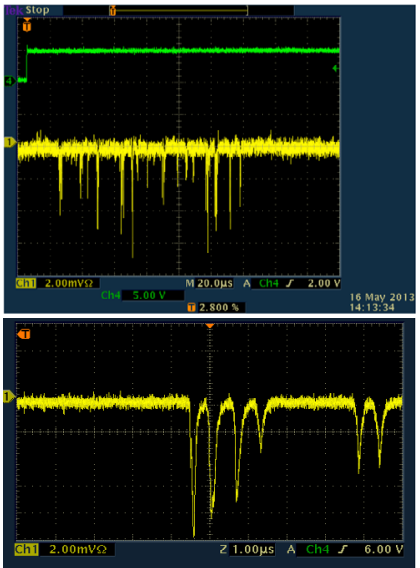

Fig. 4. Upper panel: Oscilloscope screen-shot of the detector response to an alpha particle track in propane at $400 \mathrm{~Pa}$. (Ch1) detector signal on $50 \Omega$ load; (Ch4) rising edge of the acquisition window started by the trigger. Lower panel: expanded time view of the detector signal. Measurement performed with an $\mathrm{HV}$ of $-800 \mathrm{~V}$ and an $E_{d}$ of $20 \mathrm{~V} \mathrm{~cm}^{-1}$.

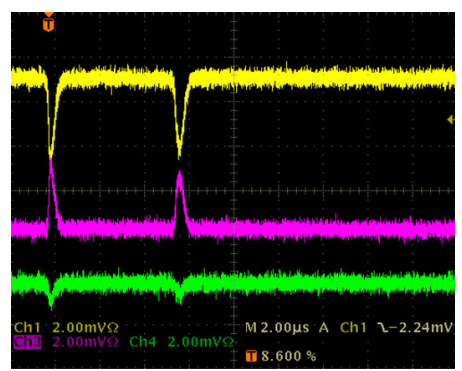

Fig. 5. Oscilloscope screen-shot displaying an expanded time view of the signal induced by two avalanches on the top (Ch1), middle (Ch3) and bottom (Ch4) layers of the detector readout strips, respectively.

resistivity glass cathode is shown in Figure 4. The signal corresponds to an alpha track in propane at a pressure of $400 \mathrm{~Pa}$. The expanded time view shows that the detector output consists of a series of negative pulses with amplitudes varying from $2 \mathrm{mV}$ to $8 \mathrm{mV}$ on $50 \Omega$ load and duration of about $500 \mathrm{~ns}$. Each pulse corresponds to an average electron avalanche charge of the order of tens of $\mathrm{pC}$. The variation in the signal amplitude indicates that the discharge region in the hole containing the avalanche is restricted and its size depends on the location along the hole axis of the initial ion-induced ionization.

Figure 5 shows the signal obtained by using the PCB with three layers of readout strips. For this measurement, the strips common to each layer were connected to ground through a $50 \Omega$ load.

Figure 6 upper panel shows the distribution of the measured ion arrival time for two different values of the applied drift voltage. As expected, the maximum of the distribution moves towards larger time values when decreasing the drift voltage. This behavior confirms that the signal registered by the detector originates from the particle track. 

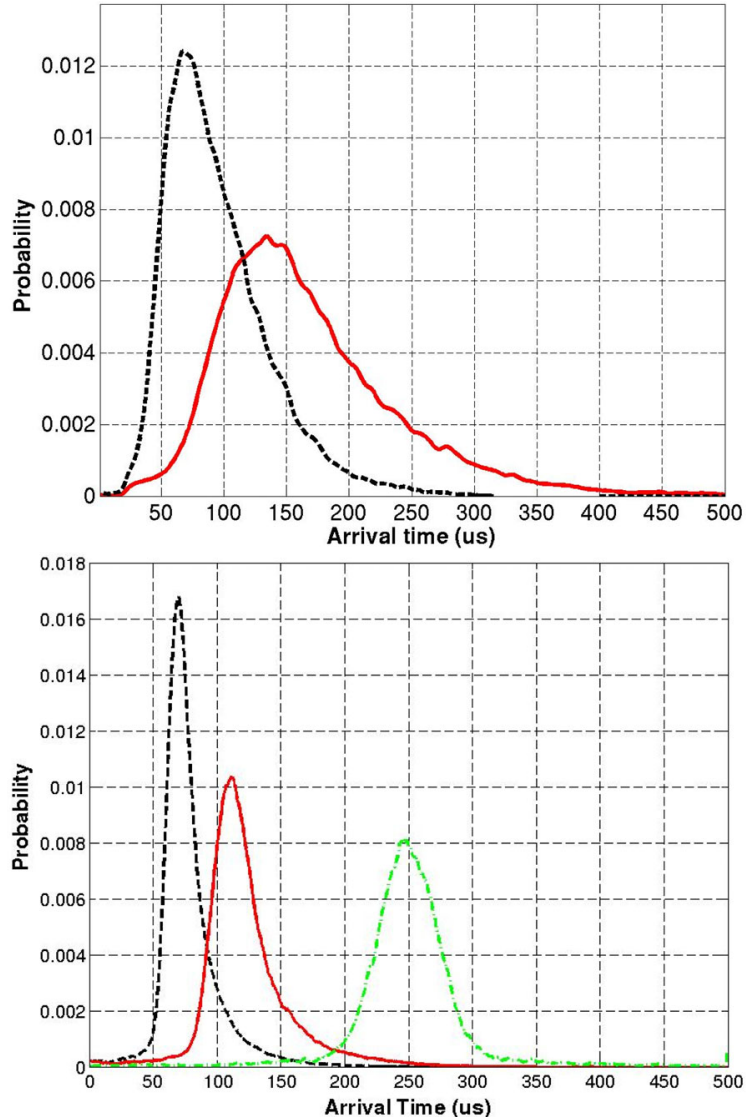

Fig. 6. Upper panel: Distribution of ion arrival time measured with the FPGA for a drift field of $20 \mathrm{~V} \mathrm{~cm}^{-1}$ (dashed line) and $10 \mathrm{~V} \mathrm{~cm}^{-1}$ (solid line). Measurements performed at a propane pressure of $400 \mathrm{~Pa}$ and applying an $\mathrm{HV}$ of $-800 \mathrm{~V}$. Lower panel: Calculated ion arrival time distribution of simulated alpha tracks. The electric field map was calculated for an $\mathrm{HV}$ of $-800 \mathrm{~V}$ and a nominal drift field of $\mathrm{V} \mathrm{cm}^{-1}$ (dashed line) and $10 \mathrm{~V} \mathrm{~cm}^{-1}$ (solid line). The dot-dashed line represents the distribution calculated for a uniform drift field of $10 \mathrm{~V} \mathrm{~cm}^{-1}$ and neglecting the penetration of the accelerating field in the drift region.

In Figure 6 lower panel, the calculated ion arrival time distributions are shown. To obtain the calculated distributions, alpha particle tacks produced in propane at $400 \mathrm{~Pa}$ were simulated with PTra, a Monte Carlo code developed at the PTB for nanodosimetry applications [11]. The coordinates of the ionization events were superimposed with the electric field map calculated with the QuickField software (http://www.quickfield.com/). The field map obtained for a drift field of $20 \mathrm{~V} \mathrm{~cm}^{-1}$ and a $\mathrm{HV}$ of $-800 \mathrm{~V}$ is shown in Figure 2. For the calculation of the drift time, a value of ion mobility of $0.793 \mathrm{~cm}^{2} \mathrm{~V}^{-1} \mathrm{~s}^{-1}$ at standard pressure and temperature was assumed [12]. This value was scaled by the ratio of the standard pressure to the pressure set in the measurements. The contribution of the ion diffusion was also included in the calculations: as obtained in [5] for similar experimental conditions, we assumed the distribution of the ion displacement along the drift direction as a Gaussian with a FWHM of about $10 \%$ of the drift distance.

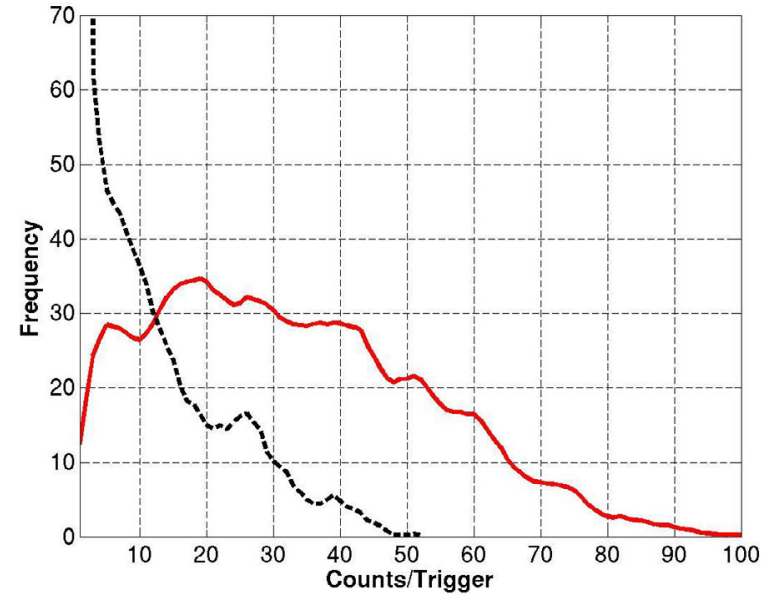

Fig. 7. Distribution of the counts per trigger registered by the detector assembled with the high-resistivity glass (dashed line, mean counts/trigger $=14$ ) and the semiconductor cathode (solid line, mean counts/trigger $=32$ ). Measurements performed at a propane pressure of $400 \mathrm{~Pa}$, an $\mathrm{HV}$ of $-800 \mathrm{~V}$ and an $E_{d}$ of $20 \mathrm{~V} \mathrm{~cm}^{-1}$.

We calculated the ion arrival time distribution for a nominal drift field of $20 \mathrm{~V} \mathrm{~cm}^{-1}$ and $10 \mathrm{~V} \mathrm{~cm}^{-1}$, and we compared the results with the measured distributions. As shown in Figure 6, both the calculated and measured distributions are characterized by a similar asymmetric shape. The calculated most frequent arrival time is in agreement with the measured most frequent value for the both drift fields. However, the measured distributions are broader than the calculated distributions; this can be caused by the presence of different species of ions created in propane, neglected in the calculations. Moreover, a more detailed description of the ion diffusion could be necessary to obtain more accurate results. The distribution of the ion interaction point in the hole can also influence the ion arrival time.

In Figure 6 lower panel, the arrival time distribution calculated in the case of a uniform drift field, i.e. neglecting the penetration of the accelerating field in the drift region, is also shown (dot-dashed line). This distribution was compared with the distribution calculated for the same drift voltage but including the penetrating field (solid line). It can be observed that the nonuniform electric field configuration present in the detector causes a shift of the distribution peak towards lower values of time and a tail at larger times.

Figure 7 shows the comparison between the distribution of the detected ions per primary particle obtained using two different detector prototypes. Both detectors were assembled with the PCB with common top electrode but differed in the cathode material (resistive glass and semiconductor). A higher ion detection efficiency was observed with the semiconductor cathode.

The ideal detector response to a beam of $4.6 \mathrm{MeV}$ alphas interacting with a volume of $2 \times 5 \times 1 \mathrm{~cm}$ of propane at $400 \mathrm{~Pa}$ was simulated, using the Geant4 toolkit [13], in order to estimate the detector efficiency. The low energy 


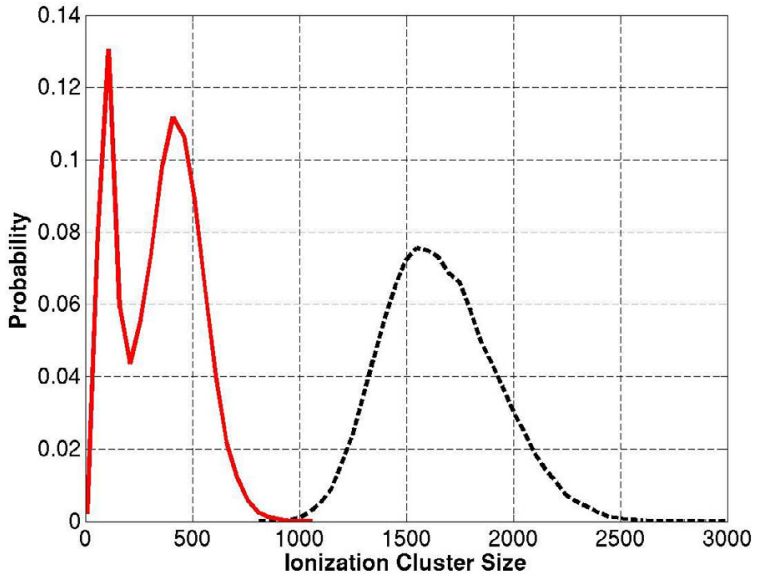

Fig. 8. Simulation of the ICSD produced by $4.6 \mathrm{MeV}$ alphas in a volume of $2 \times 5 \times 1 \mathrm{~cm}$ of $400 \mathrm{~Pa}$ propane. Ideal detector response (dashed line, mean ICS $=1655$ ). Response simulated taking the detector geometrical efficiency into account (solid line, mean ICS $=342$ ).

Livermore cross-sections for propane were used to calculate the energy deposited in the working gas and the number of ionizations produced was calculated as the ratio of the energy deposited and the $W$ value for alphas in propane $(27 \mathrm{eV}[14])$. The resulting expected mean cluster size is two orders of magnitude larger than the average number of measured counts per trigger. Figure 8 shows the comparison of the ideal ICSD and the simulated ICSD including the geometric detector efficiency (the ratio of hole area and detector area). It is apparent that up to $78 \%$ of the ions were lost due to the geometrical factor. However, the simulation ignores that the actual collection efficiency could be higher due to the focusing of ions into the holes by the penetration of the accelerating field.

The focusing can be additionally enhanced by using the readout strip layers as elements of ion optics. To this purpose, an AC readout scheme can be implemented and increasing potentials can be applied to the consecutive strip layers. In order to optimize the electric field configuration, the field map in the detector was calculated with the QuickField software varying the values of the voltage applied to the anode and to the three readout strip layers. Figure 9 shows the result of this calculation for a drift field of $20 \mathrm{~V} \mathrm{~cm}^{-1}$ and a voltage of $0 \mathrm{~V},-40 \mathrm{~V}$ and $-80 \mathrm{~V}$ applied to the top, middle and bottom layer, respectively. Comparing the electric field configuration obtained under these conditions to the one shown in Figure 2, the improved focusing can be seen.

In the case of densely ionizing radiation, e.g. alpha particles, the detector efficiency can also be reduced by the dead time. The dead time depends on the ion-charge collection, which is estimated to be of the order of $\mu$ s and on the cathode recharging time that depends on the cathode material. The higher detector efficiency observed for the detector with the semiconductor cathode could be explained by a shorter recharge time. To additionally reduce the dead time, slightly conductive PCB materials could be used in order to allow faster removal of the ions produced

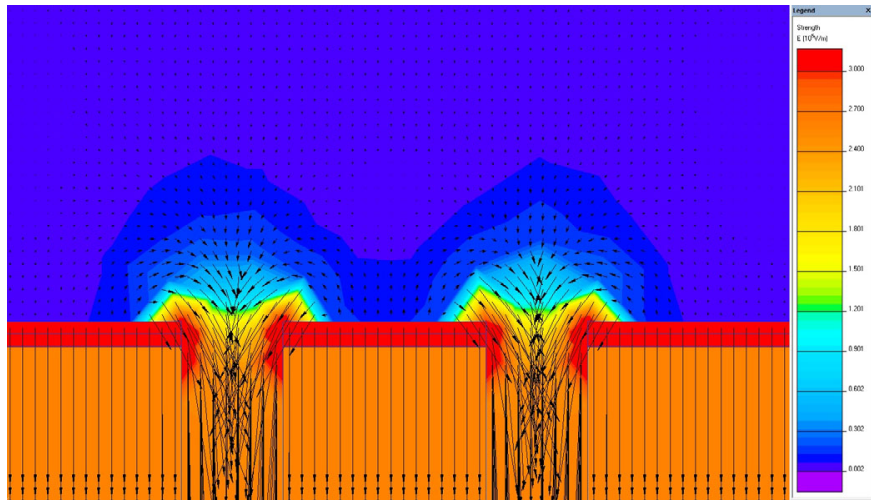

Fig. 9. Color-map representation of the electric field strength, in a vertical section of the detector, calculated with the QuickField software. The electric field lines are represented by arrows. The calculation was performed for a drift field of $20 \mathrm{~V} \mathrm{~cm}^{-1}$, a voltage of $0 \mathrm{~V},-40 \mathrm{~V}$ and $-80 \mathrm{~V}$ applied to the top, middle and bottom layer, respectively, and an $\mathrm{HV}$ of $800 \mathrm{~V}$.

in the avalanche that remain attached to the hole walls, screening the accelerating field.

The detector background noise is caused by spurious discharges and ionizations from cosmic and background radiation. The measured rate was less than $40 \mathrm{~Hz}$ for the total signal collected from all holes. However, the noise value increased when the pressure was decreased below $400 \mathrm{~Pa}$. At a pressure of $200 \mathrm{~Pa}$, the noise rate was larger than $200 \mathrm{~Hz}$. The noise rate was higher when the semiconductor cathode was used. In future experiments, different cathodes will be tested in order to find the optimal material and thickness.

\section{Conclusions}

The measurements performed in this work demonstrate that the novel ion detector can be used to register the spatial ionization pattern of alpha particles, albeit with a relatively low efficiency of a few percent. One should note that the detector is still under development and its performance can be improved. In particular, the ion collection efficiency can be enhanced by optimizing the diameter and pitch of the PCB holes and by using the readout strip layers to increase the ion focusing into the holes as shown in the simulations. The probability to produce ionimpact ionization of the working gas can be further enhanced by using a thicker PCB. The cathode material can also be optimized in order to obtain a lower recharge time and a lower noise rate. Finally, different working gases can be tested, including gas mixtures, to prevent spurious discharges.

The novel detector has potential applications in radiobiology, particle radiation therapy and radiation protection. ICSDs measured in gas could be used to benchmark Monte Carlo simulation codes that then can provide ICSDs in water or DNA. These could be used as input for radiobiological models that predict the yield of 
different types of DNA damage. This information could help to create treatment plans optimized according to DNA damage yield. This approach could also be used to predict radiation-induced cancer risk from low-dose mixed radiation fields.

This work is supported by the European ANDANTE Project funded by Euratom FP7. The PTra code was developed by Bernd Grosswendt and its latest version kindly provided by Marion Bug at the PTB.

\section{References}

1. E. Pastwa, R.D. Neumann, K. Mezhevaya, T.A. Winters, Radiat. Res. 159, 251 (2003)

2. B. Grosswend, Radiat. Prot. Dosim. 115, 1 (2005)

3. R.W. Schulte, AIP Conf. Proc. 1345, 249 (2011)

4. B. Grosswend, Radiat. Prot. Dosim. 110, 789 (2004)
5. G. Garty, S. Shchemelinin, A. Breskin, R. Chechik, G. Assaf, I. Orion, V. Bashkirov, R. Schulte, B. Grosswendt, Nucl. Instrum. Methods Phys. Res. A 491, 212 (2002)

6. S. Pszona, J. Kula, S. Marjanska, Nucl. Instrum. Methods Phys. Res. A 447, 601 (2000)

7. L. De Nardo, A. Alkaa, C. Khamphan, V. Conte, P. Colautti, P. Segur, G. Tornielli, Nucl. Instrum. Methods Phys. Res. A 484, 312 (2002)

8. V.A. Bashkirov, R.F. Hurley, R.W. Schulte, in NSS/MIC Conference Record, IEEE, 2009, Vol. 694

9. R. Chechik, A. Breskin, C. Shalem, D. Mörmann, Nucl. Instrum. Methods Phys. Res. A 535, 303 (2004)

10. R. Santonico, R. Cardarelli, Nucl. Instrum. Methods Phys. Res. A 187, 377 (1981)

11. B. Grosswend, Radiat. Environ. Biophys. 41, 103 (2002)

12. H. Raether, Electron Avalanches, Breakdown in gases, edited by J.D. Craggs, J.M. Meek (Butterworths, London, 1964)

13. GEANT4 Collaboration, Nucl. Instrum. Methods Phys. Res. A 506, 250 (2003)

14. I.K. Bronic, Radiat. Prot. Dosim. 70, 33 (1997) 